\title{
Wearable Health Devices - Updates, Applications and Repeatability
}

\author{
Khalid Ahmad Khattak \\ School of Computing and Mathematics \\ Keele University, \\ Keele, UK \\ k.a.khattak@keele.ac.uk
}

\begin{abstract}
This paper summarises PhD research into wearable health devices and outlines three planned studies 1) a study of user and stakeholder opinions of updates, 2) a performance comparison of different device models from the same device 'family' and 3) a survey of clinical trial applications with an investigation of repeatability.
\end{abstract}

Keywords: Wearable devices, health technology, clinical trials, software updates and changelogs.

\section{INTRODUCTION}

These days wearable health devices generally provide estimates for step counting, sleep tracking, stress estimation and energy expenditure and, increasingly, they also monitor heart rate. But the reliability and accuracy of these devices is an issue (Henriksen et al, 2018, Oniani et al. 2018). Updates can and do affect performance and they can be applied to:

- Operating systems

- Cloud platform algorithms

- Firmware

- Software/apps

\section{UPDATES, CHANGELOGS AND 'ITERATIVE' DEVICES}

In the research community, version reporting is important for reproducibility however, there has been only one study of consumer wearable health device changelog listings of updates (Woolley et al, 2019).

Fagan et al (2015) reported the neglect of updates in the research literature and observed that "no prior study can be found that investigated users' opinions regarding various design alternatives". Whereas Vitale et al (2017) recommended several suggestions for improvements and they are as follows:

- enabling updates to be reversible
- security updates should be released separately so that they can be installed regularly

- other updates should be available and installed selectively

- consent from the user should be required prior to implementing substantive changes in order to keep transparency

Update recommendations from practitioner software developers and user experience (UX) designers includes:

- dating of updates

- labelling of updates

- changelog maintenance

- provision of appropriate level of information to the users (Gill, 2019).

There has been little research into updates and the effects of updating wearable health devices, the styles of changelogs, and how the consumers feel about updates. This research will investigate how improvements can be made to changelog styles, updating devices and consumer satisfaction level. Also, firmware and software updates can and do change parameter estimation and device performance.

The U.S. Food and Drug Administration (FDA) 'Digital Health Software Precertification (Pre-Cert) Program' aims to achieve a more agile approach to digital health technology regulation that recognizes the "iterative characteristics" of new devices (CTA, 2018). However, there is an absence of device version reporting across the literature and its impact on the device validity after a firmware and or 
software update is installed (Collins et al, 2019). Investigating users' opinions about design of changelogs and updates might bring some fruitful results.

\section{PLANNED STUDIES}

\subsection{Study 1 - Stakeholder Opinions of Wearable Device Updates}

This study will recruit and survey healthcare providers, researchers, users and non-users of wearable health devices to investigate opinions and preferences in device changelogs and updates. For example, changes made from version 3.20 to 3.40 for Garmin Vivosmart 3 are as follows:

- Improved classifier for rep counting

- General improvements (Garmin, 2019)

Similarly, updates published in VERSION 1.49 .45 for Fitbit Charge 3 are as follows:

- You can now track runs with GPS automatically. For more information, see How do I track my workouts with my Fitbit device?

- $\quad$.....

- We added new clock faces so you can customize Charge 3 to fit your style.

- This release includes bug fixes and improvements (Fitbit, 2020).

One can observe the differences in style of presenting information to users from aforementioned examples.

\subsection{Study 2 - Performance Comparison of Different Device Models and Versions}

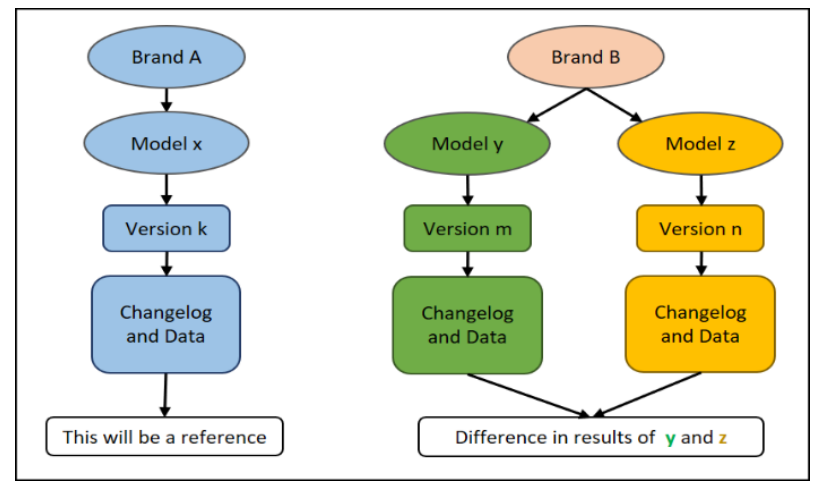

Figure 1: Overview of an experimental design to demonstrate the effects of updates and importance of model/version reporting.

Further investigation will be conducted to explore the effects of device updates, and the nature of changelog styles. Figure 1 shows one of the anticipated experiments. Here, in Figure 1, there will be three devices, one will be a reference device and the other two will be from the same family (Brand) with different models. This will identify the differences in results among these devices using the same activities. The changelogs of all devices will be checked and reported with other details of the experiment for the purpose of reproducibility and repeatability when publishing this work. This experiment will help to establish the effects of updates and the importance of changelog, accuracy, reproducibility and repeatability.

\subsection{Study 3 - Survey of Applications}

Search and explore the NIH international database of Clinical Trails (https://clinicaltrials.gov/) to observe and analyse how wearables are used and reported in clinical trials.

The following example, in Figure 2, shows the exponential rise in clinical studies using wearable health devices indicated by the number of studies found for "Fitbit" interventions in the ClinicalTrials database.

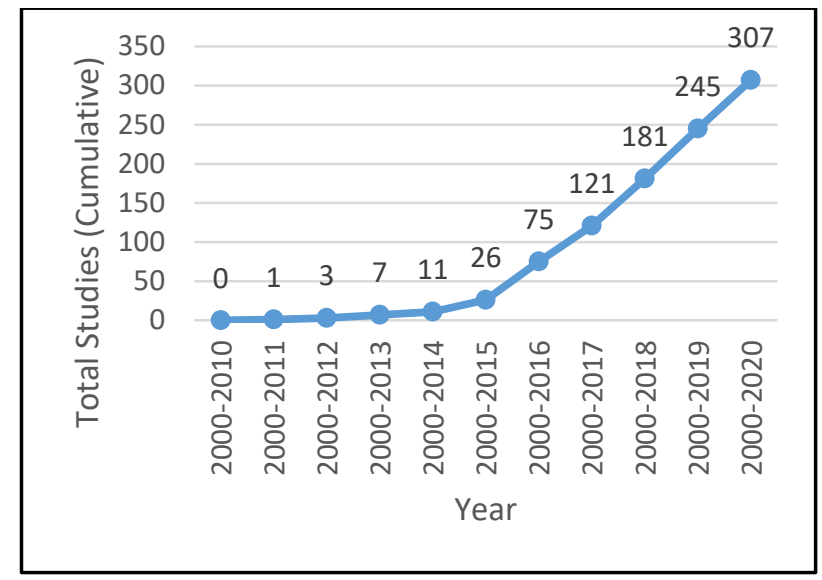

Figure 2: Total accumulated clinical studies starting during the indicated years that include "Fitbit" in intervention fields.

Similar searches will be performed with other wearable health devices' keywords to compare and evaluate the results.

\section{ABOUT THE RESEARCH}

The PhD commenced part-time in August 2019. It is supervised by Dr Sandra I. Woolley and based in The Software and Systems Engineering Research Group in the Centre for Computer Science Research at Keele University, UK.

\section{REFERENCES}

Chen J. Outstanding Release Notes Examples (And How to Use Each). (2017). https://www.appcues.com/blog/release-notesexamples (accessed February 2020) 
Collins, T., Woolley, S.I., Oniani, S., Pires, I.M., Garcia, N.M., Ledger, S.J. and Pandyan, A., (2019). Version reporting and assessment approaches for new and updated activity and heart rate monitors. Sensors, 19(7), p.1705.

CTA. CTA Announces Standard to Improve Heart Rate Monitoring in Wearables. (2018) Available online: https://www.cta.tech/News/PressReleases/2018/May/CTA-Announces-Standardto-Improve-Heart-Rate-Monit.aspx (accessed June 2020).

Fagan, M., Khan, M.M.H. and Nguyen, N., (2015). How does this message make you feel? A study of user perspectives on software update/warning message design. Human-centric Computing and Information Sciences, 5(1), p.36.

Fitbit, What's Changed in the Latest Fitbit device updates? (2019). Available online: https://help.fitbit.com/articles/en_US/Help_article /1372 (accessed June 2020)

Garmin, Updates and Downloads, Change History, (2019). Available online: https://www8.garmin.com/support/download_det ails.jsp?id=11225 (accessed June 2020)

Gill R. As a Designer I want better Release Notes. (2017). https://uxdesign.cc/design-betterrelease-notes-3e8c8c785231 (accessed February 2020)
Henriksen, A., Mikalsen, M.H., Woldaregay, A.Z., Muzny, M., Hartvigsen, G., Hopstock, L.A. and Grimsgaard, S., (2018). Using fitness trackers and smartwatches to measure physical activity in research: analysis of consumer wrist-worn wearables. Journal of medical Internet research, 20(3), p.e110.

Lacan $0 . \quad$ Keep a Changelog https://keepachangelog.com/en/1.0.0/ (accessed February 2020)

Oniani, S., Woolley, S.I., Pires, I.M., Garcia, N.M., Collins, T., Ledger, S. and Pandyan, A., (2018). Reliability assessment of new and updated consumer-grade activity and heart rate monitors, SENSORDEVICES, Venice.

Vitale, F., Mcgrenere, J., Tabard, A., BeaudouinLafon, M. and Mackay, W.E., (2017), May. High costs and small benefits: a field study of how users experience operating system Upgrades. In Proceedings of the $2017 \mathrm{CHI}$ Conference on Human Factors in Computing Systems (pp. 4242-4253).

Woolley, S., Collins, T., Mitchell, J. and Fredericks, D., (2019). Investigation of wearable health tracker version updates. BMJ Health \& Care Informatics, 26, 